\title{
Depletion of Ly6G/Gr-1 Leukocytes after Spinal Cord Injury in Mice Alters Wound Healing and Worsens Neurological Outcome
}

\author{
David P. Stirling, ${ }^{1}$ Shuhong Liu, ${ }^{1}$ Paul Kubes, ${ }^{2}$ and V. Wee Yong ${ }^{1}$ \\ ${ }^{1}$ Hotchkiss Brain Institute and Department of Clinical Neurosciences, and ${ }^{2}$ Immunology Research Group, Department of Physiology and Biophysics, \\ University of Calgary, Calgary, Alberta, Canada T2N 4N1
}

\begin{abstract}
Spinal cord injury (SCI) induces a robust inflammatory response and the extravasation of leukocytes into the injured tissue. To further knowledge of the functions of neuroinflammation in SCI in mice, we depleted the early arriving neutrophils using an anti-Ly6G/Gr-1 antibody. Complete blood counts revealed that neutrophils increased $\sim 3$-fold over uninjured controls and peaked at $6-12 \mathrm{~h}$ after injury, and that anti-Ly6G/Gr-1 treatment reduced circulating neutrophils by $>90 \%$ at these time points. Intravital and spinning disk confocal microscopy of the exposed posterior vein and postcapillary venules showed a significant reduction in rolling and adhering neutrophils in vivo after anti-Ly6G/Gr-1 treatment; this was accompanied by a parallel reduction in neutrophil numbers within the injured spinal cord at 24 and $48 \mathrm{~h}$ as determined by flow cytometry. The evolution of astrocyte reactivity, a wound healing response, was reduced in anti-Ly6G/Gr-1-treated mice, which also had less spared white matter and axonal preservation compared with isotype controls. These histological outcomes may be caused by alterations of growth factors and chemokines important in promoting wound healing. Importantly, anti-Ly6G/Gr-1 treatment worsened behavioral outcome as determined using the Basso Mouse Scale and subscores. Although the spectrum of cells affected by anti-Ly6G/Gr-1 antibody treatment cannot be fully ascertained at this point, the correspondence of neutrophil depletion and worsened recovery suggests that neutrophils promote recovery after SCI through wound healing and protective events that limit lesion propagation.
\end{abstract}

Key words: spinal cord injury; neuroinflammation; neutrophils; microglia; astrocyte; neurotrophic factors

\section{Introduction}

Spinal cord injury (SCI) elicits a complex cascade of events that further promote tissue destruction and ultimately results in poor clinical prognosis. This cascade, known as secondary degeneration, involves vascular disturbances, ischemia/hypoxia, propagation of free radicals, excitotoxicity, ionic dysregulation, and inflammation, which leads to necrotic and apoptotic death of neurons and glia, and subsequent demyelination and axonal damage (Dumont et al., 2001; Kwon et al., 2004). Although the initial mechanical destructive events cannot be reversed, secondary degeneration occurs over several hours to weeks, a time frame during which therapeutic intervention may be achieved.

Similar to other injured tissues, an inflammatory response ensues after SCI, marking the beginning of wound healing and

\footnotetext{
Received 0ct. 13, 2008; revised Nov. 15, 2008; accepted Nov. 30, 2008.

This work was supported by grants from the Canadian Institutes of Health Research and NeuroScience Canada. D.P.S. was funded by a fellowship provided by the Alberta Heritage Foundation for Medical Research (AHFMR). P.K. and V.W.Y. are both Canada Research Chair (Tier 1) holders and AHFMR Scientists. We are indebted to Brandie Millen, University of Calgary Live Cell Imaging Facility, for her excellent technical assistance with intravital and spinning disk confocal microscopy. We are also indebted to Karen Cummins and Shuzhen Meng for their help with behavioral scoring, sample collection and tissue processing. Last, we thank both Laurie Kennedy and Laurie Robertson from the University of Calgary Flow Cytometry Core Facility for excellent advice and technical assistance.

Correspondence should be addressed to Dr. V. Wee Yong, University of Calgary, 3330 Hospital Drive, Calgary, Alberta, Canada T2N 4N1. E-mail: vyong@ucalgary.ca.

D01:10.1523/JNEUROSCI.4918-08.2009

Copyright $\odot 2009$ Society for Neuroscience $\quad$ 0270-6474/09/290753-12\$15.00/0
}

scarring events. These early responses to injury include the activation of endogenous CNS cells, microglia and astrocytes, that produce proinflammatory mediators such as cytokines, chemokines, and lipid mediators that drive leukocyte recruitment to the injured tissue (Bartholdi and Schwab, 1997; Pineau and Lacroix, 2007; Rice et al., 2007). Although the precise signals are yet unknown, SCI leads to a rapid and marked mobilization of circulating neutrophils within the first few hours after injury and their entry into the injured spinal cord that peaks at $12-24 \mathrm{~h}$ (Dusart and Schwab, 1994; Carlson et al., 1998; Stirling and Yong, 2008). Subsequently, monocytes are mobilized and recruited, followed by lymphocytes, although species and strain differences have been documented (Sroga et al., 2003; Fleming et al., 2006; Donnelly and Popovich, 2008; Stirling and Yong, 2008).

Because of their potential to release several neurotoxic substances within the injured spinal cord parenchyma, neutrophils have become an attractive target to ameliorate secondary degeneration after SCI. However, despite much work in this area, controversy ensues. Some studies that target neutrophil trafficking after SCI report a detrimental role for neutrophils (Taoka et al., 1997; Bao et al., 2004; Gris et al., 2004), but this result was not reproduced by others (Holtz et al., 1989; Forbes et al., 1994; Bartholdi and Schwab, 1995). One confounder is that inhibitory therapies used previously likely altered functions of other inflammatory subsets such as macrophages. Thus, the roles of neutro- 
phils in mediating injury or repair within the injured spinal cord remain elusive.

To further knowledge of the neutrophil response to SCI, we have applied an anti-Ly6G/Gr-1 antibody that is very potent at depleting neutrophils after a T9/10 contusion injury to mice. Although this antibody may have affinity for Ly6C that is expressed on subpopulations of monocytoid cells and lymphocytes (Fleming et al., 1993; Daley et al., 2008), treatment in our study reduced neutrophil numbers by $>90 \%$ without obvious alteration of monocyte or lymphocyte numbers. Unexpectedly, the anti-Ly6G/Gr-1-treated mice displayed worsened neurological outcome after SCI and wound healing events were impeded. These data caution against potential therapeutic strategies that inhibit neutrophils from responding to and entering the lesioned spinal cord to reduce the neurologic sequelae of SCI.

\section{Materials and Methods}

Spinal cord injury. All experiments were conducted in accordance with the University of Calgary Animal Care Ethics Committee, adhering to guidelines of the Canadian Council on Animal Care. Adult CD-1 (Charles River Laboratories) and Lysozyme-eGFP (Lyse-GFP, in which myeloid cells express green fluorescent protein) (Faust et al., 2000) mice 6-8 weeks old were anesthetized with ketamine-xylazine (85 and 15 $\mathrm{mg} / \mathrm{kg}$, respectively) and subjected to a moderate (60 kdyn) T9/10 contusion SCI (Infinite Horizons Impactor, Precision Systems) as previously described (Stirling and Yong, 2008). Before and after injury, the mice were allowed to recover in temperature-controlled incubators and received $0.5 \mathrm{ml}$ of saline to compensate for loss of blood and dehydration, and buprenorphine $(0.05 \mu \mathrm{g} / \mathrm{g})$ as an analgesic. Bladders were manually expressed two to three times daily until spontaneous voiding returned.

Treatment with anti-Ly6G/Gr-1 antibody. Functional grade purified anti-mouse Ly6G/Gr-1 (clone RB6-8C5, \#16-5931) or isotype-matched control antibodies (IgG2b isotype control, \#16-4031) were purchased from eBioscience. Animals were randomized into sham, injured treated, or controls and received either $4 \mathrm{mg} / \mathrm{kg}$ intraperitoneal injections of anti-LyG6/Gr-1 or isotype control at 2 and $24 \mathrm{~h}$ after SCI.

The RB6-8C5 clone binds to the myeloid differentiation antigen Gr-1, a glycosylphosphatidyl inositide-linked protein. Gr-1 was subsequently identified to be a member of the Ly6 gene family. Ly6G is the major antigen on mature neutrophils identified by the RB6-8C5 clone (Hestdal et al., 1991; Fleming et al., 1993); the antibody depletes neutrophils in both blood and spleen for up to 2-3 d after injection (Conlan and North, 1994; Rakhmilevich, 1995). The RB6-8C5 anti-Ly6G/Gr-1 antibody, however, has been reported to bind to Ly6C, which is found on a subset of monocytoid cells and lymphocytes (Fleming et al., 1993; Daley et al., 2008), although this cross-reactivity was not observed by others (Nagendra and Schlueter, 2004).

Harvesting of tissue and blood. At several time points after SCI, animals were anesthetized with a lethal dose of ketamine-xylazine, and $0.5 \mathrm{ml}$ of whole blood was collected by cardiac puncture into $100 \mu \mathrm{l}$ of heparin solution (Hepalean, Organon). Immediately after blood collection, the animals were perfused with ice-cold PBS and the spinal cord $(2 \mathrm{~cm}$ centered around the lesion site) was rapidly isolated and placed into ice-cold PBS. Spinal cord samples were then prepared for flow cytometry. Naive or sham-injured animals served as controls.

Flow cytometry. Spinal cord samples were prepared for flow cytometry as previously described (Stirling and Yong, 2008). Briefly, the spinal cord samples were mechanically disrupted and passed through a wire mesh screen (Sigma-Aldrich). Samples were then subjected to centrifugation and resuspended in fetal bovine serum (FBS) staining buffer (BD Biosciences). Samples were divided, and cells alone and isotype-matched control samples were generated to control for nonspecific binding and autofluorescence. Isotype control antibodies were purchased from BD Biosciences PharMingen and included phycoerythrin (PE)-labeled rat IgG2b, $\kappa$, FITC-labeled rat IgG2b, $\kappa$, and peridinin chlorophyll-a protein (PerCP)-conjugated rat IgG2b, $\kappa$. After cell counts, the samples were given Fc block (BD Biosciences) to minimize background staining. The following antibodies were purchased from BD Biosciences PharMingen, and were optimized and used to determine populations of resident microglia and blood-derived leukocytes: $R$-phycoerythrin-conjugated rat anti-mouse CD11b, FITC anti-mouse Ly-6G and Ly-6C (Gr-1), PerCPconjugated rat anti-mouse CD45 (leukocyte common antigen, Ly-5). After 30 min of incubation with combinations of antibodies at $4^{\circ} \mathrm{C}$, the samples were washed and fixed in $1 \%$ buffered formalin. The samples were then immediately analyzed with a Becton Dickinson LSR Benchtop Flow Cytometer (BD Biosciences). Density plots were created using WinMDI version 2.8 software (Scripps Research Institute), and cells alone and isotype controls were used to set quadrants as previously described (Stirling and Yong, 2008). CD45-positive gates ensured that only leukocytes and resident microglia were included in the analysis. The proportion of blood-derived leukocytes (neutrophils and monocytes) $\left(\mathrm{CD} 45^{\text {high }}: \mathrm{CD} 11 \mathrm{~b}\right)$, neutrophils (CD45 $\left.{ }^{\text {high }}: \mathrm{Gr}-1^{\text {high }}\right)$, and resident microglia (CD $45^{\text {low }}$ :CD11b) was determined from density plots (CD45, $y$-axis; CD11b or Gr-1, $x$-axis) using WinMDI version 2.8 software (Scripps Research Institute) and compared between the different groups. The CD $45^{\text {high }}:$ Gr- $1^{\text {high }}$ neutrophils were previously shown to be negative for F4/80, CD107b, or CD3, discriminating them from monocytoid cells (F4/80 and CD107b positive) or T lymphocytes (CD3+) (Stirling and Yong, 2008).

Complete blood counts. Complete blood counts were performed as previously described (Stirling and Yong, 2008). Briefly, at 0, 6, 12, or $48 \mathrm{~h}$ after SCI, animals ( $n=3-6$ per group, per time point) were anesthetized with a lethal dose of ketamine-xylazine, and $0.5 \mathrm{ml}$ of whole blood was collected by cardiac puncture into BD Microtainer tubes with EDTA (BD Biosciences). The samples were immediately analyzed using a COULTER Gen-S Hematology Analyzer (BD Biosciences), and blood smears were prepared and white blood cell differential counts were performed by a clinical unit, Calgary Laboratory Services.

Intravital microscopy of spinal vasculature. Intravital microscopy of the mouse posterior vein and postcapillary venules was performed in vivo at $0,15 \mathrm{~min}$, and 6 and $12 \mathrm{~h}$ after injury. Mice were anesthetized as above and the tail vein or jugular vein was cannulated for the administration of additional anesthetic and fluorescent dyes. The skin and overlying musculature was reopened to expose the spinal cord vasculature, and the spinal column was stabilized using forceps mounted on a stage to reduce breathing artifacts. The mouse was maintained at $37^{\circ} \mathrm{C}$ and the exposed spinal cord was kept moist with an artificial CSF buffer. Circulating leukocytes were fluorescently labeled by intravenous administration of rhodamine $6 \mathrm{G}$ (Sigma-Aldrich; $0.5 \mathrm{mg} / \mathrm{kg}$ of body weight). Labeled cells were observed using an IV500L Mikron epifluorescence microscope and outfitted with a fluorescent light source (epi-illumination at $510-560 \mathrm{~nm}$ using a $590 \mathrm{~nm}$ emission filter). A low light intensifier charge-coupled device (ICCD) camera (Stanford Photonics) mounted to the microscope was used to project the image to a monitor. All experiments were recorded for later analysis. Assessment of rolling and adherent leukocytes was performed as previously described (Kerfoot et al., 2006). In brief, rolling leukocytes were defined as those cells moving at a velocity less than that of erythrocytes within a given vessel. Rolling flux was determined as the number of rolling cells that pass a particular point in a vessel over the course of a minute. Leukocytes were considered adherent if they remained stationary for $30 \mathrm{~s}$ or longer.

Spinning disk confocal microscopy of spinal vasculature. Exposure of the spinal vasculature was performed as described above for intravital microscopy. Images and video of the exposed posterior vein and postcapillary venules were acquired with an Olympus BX51 upright microscope using a $20 \times$ air objective as previously described (Norman et al., 2008). The microscope was equipped with a confocal light path (WaveFx, Quorum) based on a modified Yokagawa CSU-10 head (Yokagawa Electric Corporation). After laminectomy (sham controls) or after SCI at $6 \mathrm{~h}$, $48 \mathrm{~h}$, and $5 \mathrm{~d}$, neutrophils were visualized in vivo after intravenous administration of anti-mouse Ly6G/Gr-1 (BD Biosciences) conjugated with APC, endothelial cells with anti-mouse CD31 conjugated to FITC, and monocytes with anti-mouse Ly6C-PE antibody simultaneously using the appropriate filters. A back-thinned EMCCD camera (C9100, Hamamatsu) was used for fluorescence detection. Images captured using 
the spinning disk were processed and analyzed using Volocity Acquisition software (Improvision).

Histology and immunohistochemistry. At several time points after SCI, animals were anesthetized with a lethal dose of ketamine-xylazine and perfused with ice-cold PBS, followed by perfusion fixation with a solution of $4 \%$ paraformaldehyde in PBS. The spinal cords (brainstem to lumbar region) were removed and postfixed in $4 \%$ paraformaldehyde overnight and subsequently cryoprotected in $30 \%$ sucrose. The spinal cords were cut into blocks, frozen, and stored at $-80^{\circ} \mathrm{C}$ until sectioned. Specifically, a $2 \mathrm{~cm}$ lesion block, centered at the lesion site, was cryosectioned at a thickness of $10 \mu \mathrm{m}$, and tissue sections were collected on Superfrost slides (Fisher Scientific) organized into five adjacent section series. Fluorescent immunohistochemistry was performed on slides containing sections of naive, sham, 24 and $48 \mathrm{~h}, 5 \mathrm{~d}$ and 4 week postinjured spinal cords. Slides were rinsed in PBS, blocked in $10 \%$ normal goat serum in PBS for $30 \mathrm{~min}$ at room temperature. To detect blood-derived leukocytes and resident microglia cells, the following primary antibodies were used: rabbit anti-mouse Iba-1 (1:1000, Wako Chemicals) for microglia/macrophages, and rat anti-mouse neutrophils (clone 7/4, 1:500, Serotec) for neutrophils. To detect axons, astrocytes, and fibronectin, the following primary antibodies were used: rabbit anti-neurofilament $\mathrm{H}$ $200 \mathrm{kDa}$ (1:500, Sigma-Aldrich), mouse anti-GFAP (1:1000, SigmaAldrich), anti-fibronectin (1:600, Sigma-Aldrich). Slides were then washed three times in PBS and incubated with the appropriate secondary antibody: Cy3-conjugated antibody (Jackson ImmunoResearch Laboratories), Alexa Fluor 488 antibody (Invitrogen), or Alexa Fluor 633 (Invitrogen) to visualize the primary antibodies listed above. After three washes in PBS, slides were submerged in Hoechst 33258 ( $1 \mu \mathrm{g} / \mathrm{ml}$, SigmaAldrich) to further assess polymorphonuclear cells. Images were captured using a confocal microscope equipped with a digital camera.

Residual white and gray matter. To assess sparing of white and gray matter, transverse sections were stained with a modified eriochrome cyanine (EC) protocol as previously described (Rabchevsky et al., 2001). Briefly, air-dried sections were cleared and hydrated before being placed for $10 \mathrm{~min}$ into a solution consisting of $2 \mathrm{ml}$ of $10 \% \mathrm{FeCl}_{3}$ and $40 \mathrm{ml}$ of $0.2 \%$ EC (Sigma-Aldrich) in $0.5 \%$ aqueous $\mathrm{H}_{2} \mathrm{SO}_{4}$ brought to a final volume of $50 \mathrm{ml}$ with $\mathrm{dH}_{2} \mathrm{O}$. This was followed by washes in water and differentiation for $10 \mathrm{~s}$ in $0.5 \%$ aqueous $\mathrm{NH}_{4} \mathrm{OH}$. The reaction was terminated with rinses in water before slides were immersed in $1 \%$ neutral red solution for $1 \mathrm{~min}$. Slides were then washed in $\mathrm{dH}_{2} \mathrm{O}$, dehydrated, cleared, and mounted. Images were captured with a bright-field microscope ( $4 \times$ magnification). The section with the least amount of spared white matter was designated as the lesion epicenter. Spared white matter, lesion area, and total cross-sectional area were measured using SigmaScan Pro Version 5 image analysis software (Systat Software) or Image J 1.37J software (National Institutes of Health, Bethesda, MD) by a person blinded to treatment and reported as a percentage of the total crosssectional area (Basso et al., 2006).

Neurofilament and Iba-1 quantification. To assess axonal sparing within the ventral and lateral columns within the lesion epicenter, an overlay of one rectangular box (100 $\mu \mathrm{m}$ width and $600 \mu \mathrm{m}$ length) was placed onto digitally captured sagittal sections containing neurofilament 200 (NF200)-labeled axons (green channel) using SigmaScan Pro Version 5 software (Systat Software). With regard to the placement of the rectangle, the ventral-most edge of the midline of the spinal cord was set at $0 \mu \mathrm{m}$. The rectangle was placed so that its top edge was aligned $400 \mu \mathrm{m}$ from the midline and captured an area $\left(60,000 \mu \mathrm{m}^{2}\right)$. The overlay of the rectangle was then copied onto thresholded images depicting the NF200positive profiles (green channel), and the percentage of the area occupied by NF200 signal was reported. Three sections from 11 to 12 animals per group were assessed. Similarly, Iba-1 density within the lesion epicenter was quantified using the same approach with a standardized overlay box (250 $\mu \mathrm{m}$ width, $750 \mu \mathrm{m}$ length) positioned within the center of the cord. At least three sections from four animals per group were assessed.

Cytokine and growth factor quantification. To assess cytokine and growth factor levels from injured spinal cord tissue, we isolated a $1 \mathrm{~cm}$ piece of spinal cord from anti-Ly6G/Gr-1-treated, isotype, or sham animals at $48 \mathrm{~h}$ and $5 \mathrm{~d}$ after injury. For cytokine analysis, spinal cord tissue isolated from sham, anti-Ly6G/Gr-1-treated, and isotype controls were sent to Ray Biotech for protein extraction and quantification using RayBio mouse G Series inflammation Array I antibody arrays. Forty inflammatory proteins were analyzed and normalized to positive controls and reported as mean protein levels (arbitrary units) per group. Four animals from each group were assessed. For growth factor analysis, spinal cord tissue isolated from sham, anti-Ly6G/Gr-1-treated, and isotype controls were rapidly frozen and placed in TRIzol (Invitrogen) for subsequent RNA isolation and purification following the manufacturer's instructions. Quantitative RNA expression analysis of 84 growth factor-related genes was simultaneously analyzed using mouse Growth Factors $\mathrm{RT}^{2}$ Profiler PCR Array (catalog \#PAMM-041; SABiosciences) following the manufacturer's instructions. Four to five animals per group were assessed. RNA from each mouse was separately applied to each PCR template, and the threshold cycle number for each of 84 genes was normalized to four built-in housekeeping gene controls using the RT ${ }^{2}$ Profiler PCR Array Data Analysis web-based software (SABiosciences Corp.). The normalized value for each growth factor per mouse was then averaged across the four to five mice per group, and reported as fold changes over similar values from sham controls.

Wound healing and glial scar quantification. To assess glial scar formation within the ventral and lateral columns within the lesion epicenter, six rectangular boxes ( 300 pixels width and 100 pixels length) were placed laterally to medially onto a $100 \mu \mathrm{m}$ grid overlay onto digitally captured $(400 \times)$ cross-sections of GFAP (astrocytes)- and fibronectin (lesion)stained spinal cord sections. With respect to the placement of the boxes, the medial edge of the third boxes was adjacent to the glial scar wound boundary. Therefore, the six boxes sequentially capture the glial scar from the most laterally placed box (no scar) to the 3 box (maximal scar) to the last 3 boxes which contain very few astrocytes but dense fibronectin staining. Utilizing SigmaScan Pro Version 5 software (Systat Software), which thresholded images depicting the GFAP-positive profiles (red channel) and fibronectin-positive profiles (green channel), the mean percentage of the area occupied by each signal within each box was reported. Similarly, the area occupied by fibronectin staining within the center of the lesion site was also measured within a $100 \times 100$ pixel box. Three representative areas per section were measured, over three sections per animal and at least four animals per group were assessed.

Behavioral analysis. To assess functional recovery of animals after SCI, mice were tested using the open-field Basso Mouse Scale (BMS) and subscore (Basso et al., 2006). Animals were assessed before injury to ensure that no deficits in hindlimb function were evident and to expose the animals to an open-field testing environment. After injury, animals were tested on the first day, third day, and once per week thereafter until the end of the study by two blinded observers. The mean scores per treatment group were plotted as a function of time postinjury.

Statistics. Statistical analysis was performed using SigmaStat software. For behavioral analysis, a repeated-measures ANOVA was used to determine differences between the groups. For complete blood count, flow cytometry, cytokine, and growth factor analysis, groups were tested using an ANOVA and the Holm-Sidak post hoc analysis when warranted. In the case of unequal variances, the Kruskal-Wallis one way ANOVA on ranks was used. Differences with a $p$ value $<0.05$ were considered significant.

\section{Results}

\section{Anti-Ly6G/Gr-1 antibody treatment markedly reduces circulating neutrophils after SCI}

Because SCI induces a rapid and robust neutrophil response, we applied the RB6-8C5 anti-Ly6G/Gr-1 antibody in an attempt to deplete neutrophils after a T9/10 moderate (60 kdyn) contusion injury to adult mice. To determine the efficacy of neutrophil depletion, blood was isolated via cardiac puncture at several time points after SCI, smears were prepared, and complete and differential blood counts were performed by a clinical laboratory. In agreement with our previous study (Stirling and Yong, 2008), circulating neutrophils with characteristic multilobed nuclei were increased $\sim 2$-to 3 -fold over uninjured (naive) controls at 6-12 h after injury, with return to normal values by $48 \mathrm{~h}$ (Fig. 1A). Importantly, anti-Ly6G/Gr-1 treatment $(4 \mathrm{mg} / \mathrm{kg}, 2$ and 
$24 \mathrm{~h}$ after SCI) reduced absolute neutrophil counts by 91 and $96 \%$ at 6 and 12 h, respectively, compared with isotypetreated animals (Fig. 1A). This pronounced neutrophil depletion in blood was evident up to at least $48 \mathrm{~h}$ after injury (Fig. 1A).

Circulating lymphocyte numbers were significantly reduced at all time points after SCI compared with uninjured controls, but their numbers as well as those for monocytes were not obviously altered by anti-Ly6G/ Gr-1 antibody treatment (Fig. 1B,C). As well, red blood cell $(p=0.379)$ and platelet counts $(p=0.421)$ were unchanged by treatment or injury (data not shown). Absolute white blood cell counts in blood were significantly $(p<0.001)$ reduced after antiLy6G/Gr-1 treatment (Fig. $1 D$ ), likely reflecting the fact that neutrophils account for the majority of white blood cells (WBCs) acutely after SCI.

Real-time imaging in vivo reveals that anti-Ly6G/Gr-1 antibody treatment decreases leukocyte rolling and firm adhesion at 6 and $12 \mathrm{~h}$ after SCI

To further knowledge of the onset of leukocyte trafficking to the injured spinal cord and to gain further insights into the effectiveness of the anti-Ly6G/Gr-1 treatment, we performed intravital microscopy of dorsally exposed vessels within the lesion site in vivo. Leukocytes were visualized after intravenous injection of rhodamine $\mathrm{G}$, and rolling leukocytes interacting with blood vessel walls with velocities slower than those of erythrocytes (Kerfoot et al., 2006) were rarely seen within the exposed posterior vein and postcapillary venules of sham animals. Thus, laminectomy alone does not invoke a noticeable inflammatory response in control animals (supplemental video 1, available at www.jneurosci.org as supplemental material; Fig. 2). Typically, free flowing leukocytes traveled at velocities of $\sim 900-1100 \mu \mathrm{m} / \mathrm{s}$ in exposed spinal cord vasculature, similar to what has been reported for lymphocytes (Vajkoczy et al., 2001). Occasionally, leukocytes could be seen slowing down and rolling along the endothelial lumen, but firm adhesion (stationary for $30 \mathrm{~s}$ or longer) was a rare event in uninjured spinal cord vasculature (Fig. $2 A$; supplemental video 1, available at www. jneurosci.org as supplemental material). In contrast, several rolling cells were evident as early as 15 min after SCI (supplemental video 1, available at www.jneurosci.org as supplemental material). By 6 and $12 \mathrm{~h}$ after injury, many leukocytes were rolling in isotype controltreated animals; these numbers were greatly reduced in anti-Ly6G/ Gr-1-treated animals (Fig. 2B, C; supplemental video 2, available at www.jneurosci.org as supplemental material). Similarly, firm adhesion was also significantly reduced in anti-Ly6G/Gr-1-treated animals versus controls (Fig. $2 B, C$ ).

We next used spinning disk confocal microscopy to visualize fluorescently labeled cells interacting with PECAM-1/CD31labeled endothelium after SCI in vivo. As shown in Figure $2 D$ and supplemental video 2 (available at www.jneurosci.org as supplemental material), the majority of leukocytes were double positive for rhodamine $\mathrm{G}$ and Gr-1 at $6 \mathrm{~h}$ after SCI. Because only rare Ly6C cells were observed (data not shown), the results indicate
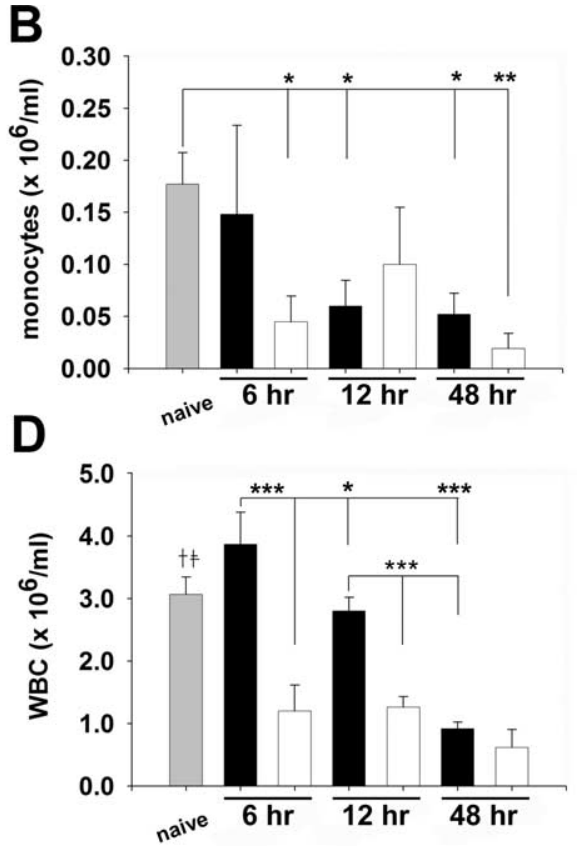

anti-Ly6G/Gr-1

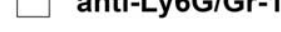

naive $\square$ isotype

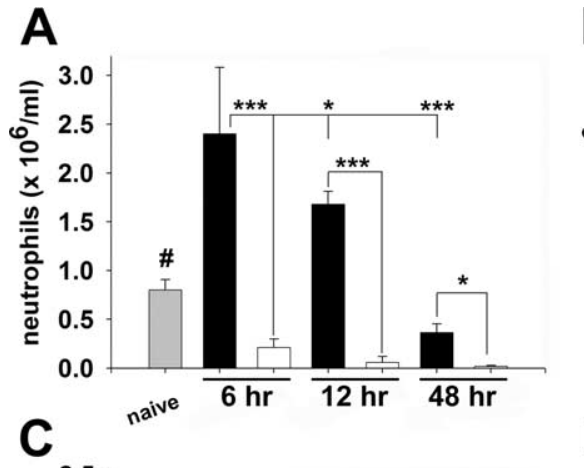

Figure 1. $A-D$, Anti-Ly6G/Gr-1 treatment reduces circulating neutrophils after $\mathrm{SCl}$. $A$, Absolute neutrophil counts from whole blood collected from naive (uninjured; gray bar) mice and at 6, 12, and $48 \mathrm{~h}$ after $\mathrm{SCl}$ reveal a pronounced neutrophilia peaking at (isotype animals; black bars in graphs). Anti-Ly6G/Gr-1 treatment (white bars) markedly $(p<0.001$, ANOVA) C), but their numbers were not significantly altered by anti-Ly6G/Gr-1 treatment. WBC counts were reduced in neutrophildepleted animals $(\boldsymbol{D})$. Data are represented as mean $\times 10^{6} \mathrm{cell} / \mathrm{s} / \mathrm{ml} \pm \mathrm{SEM}$, ANOVA with Holm-Sidak multiple comparisons test. ${ }^{\#} p<0.05$ naive vs all groups except isotype $48 \mathrm{~h}(\boldsymbol{A}) .{ }^{\dagger} p<0.001$ naive vs all anti-LyG6/Gr- 1 groups and ${ }^{\ddagger} p<0.001$ naive vs isotype $48 \mathrm{hSCl}(\boldsymbol{D}) .{ }^{*} p<0.05,{ }^{* *} p<0.01,{ }^{* * *} p<0.001, n=3-6$ per group, per time point.

that the majority of the endothelial interacting leukocytes were indeed neutrophils at these early time points. At $48 \mathrm{~h}$ after injury, anti-Ly6G/Gr-1 treatment greatly reduced the number of Ly6G/ Gr-1-positive cells compared with isotype controls (Fig. 2 E; supplemental video 3, available at www.jneurosci.org as supplemental material). Interestingly, although rolling and firm adhesion of leukocytes to endothelium was still evident at $48 \mathrm{~h}$ after injury in isotype controls, few leukocytes were interacting with endothelial cells in anti-Ly6G/Gr-1-treated animals (Fig. $2 E$ middle panel; supplemental video 3, available at www.jneurosci.org as supplemental material). At $5 \mathrm{~d}$ after injury, few fluorescent-tagged cells were present in anti-Ly6G/Gr-1-treated mice (data not shown), but many were interacting and rolling along blood vessel lumina in isotype control animals (supplemental video 4 , available at www.jneurosci.org as supplemental material).

Thus, direct visualization within spinal cord vasculature reveals a robust recruitment of leukocytes to the injury site as early as $15 \mathrm{~min}$ after SCI, where they roll and adhere to the lumen of postcapillary venules and venules feeding into the dorsally exposed posterior vein up to at least $5 \mathrm{~d}$ after injury. Importantly, anti-Ly6G/Gr-1 treatment greatly reduced the number of leukocytes available to roll and firmly adhere to endothelial cells, a prerequisite for subsequent diapedesis into most inflamed tissues.

\section{Anti-Ly6G/Gr-1 antibody treatment markedly reduces neutrophil number within the spinal cord at 24 and $48 \mathrm{~h}$ after SCI}

To further establish the efficacy of the Ly6G/Gr-1-depleting strategy, we quantified the neutrophil response within the injured 


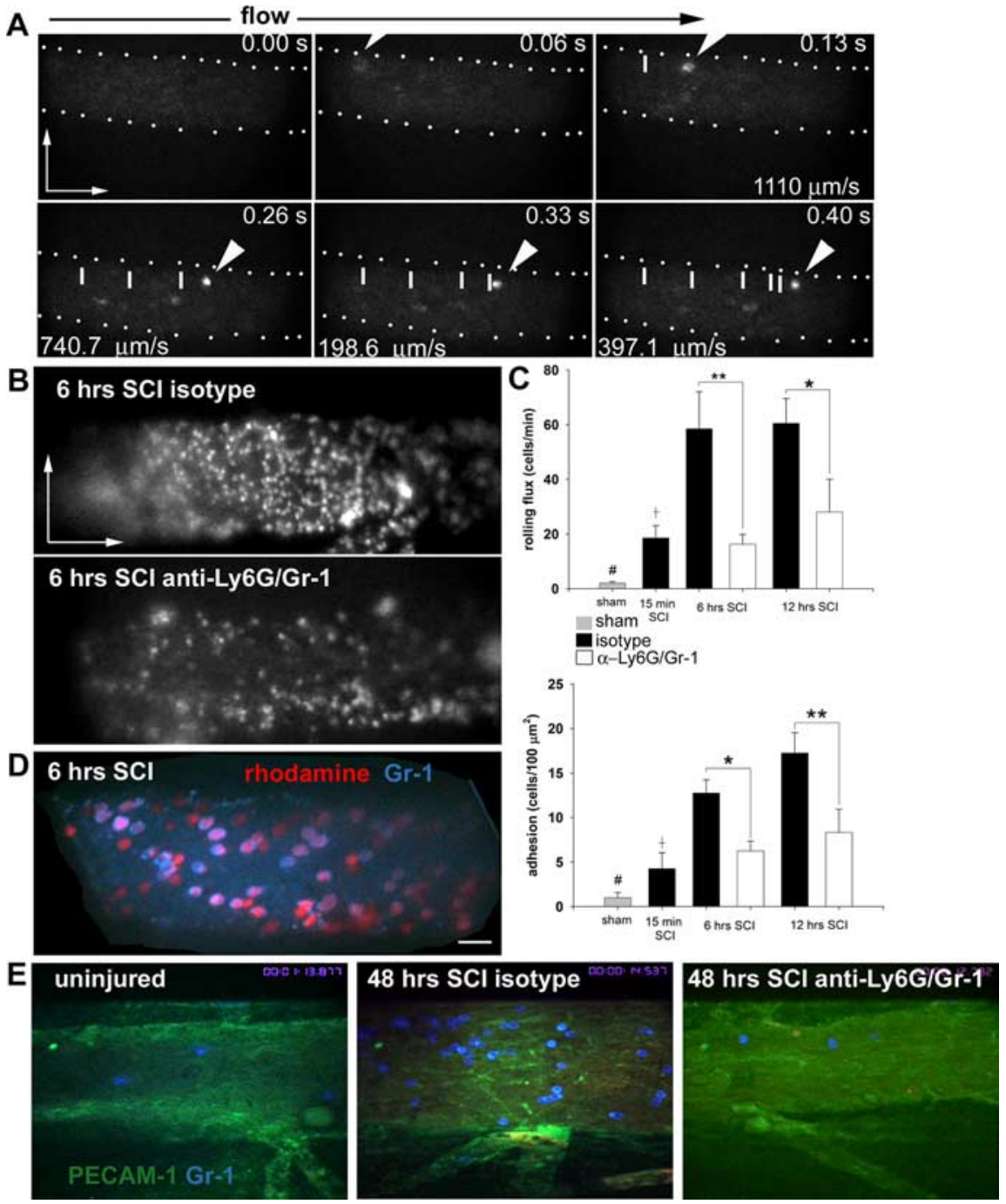

Figure 2. Anti-Ly6G/Gr-1 treatment reduces neutrophil rolling and adhesion on spinal vasculature after $\mathrm{SCl}$. $\boldsymbol{A}$, Micrographs collected from a time-lapse recording of exposed spinal cord vasculature from an uninjured (sham) mouse revealing a rhodamine G-labeled leukocyte (white arrows) slowing down and rolling along a blood vessel lumen over time (seconds) is shown in the top right corner and velocity (micrometers per second) in the bottom left corner of images. Few leukocytes are seen rolling in uninjured (sham) animals. Scale bar, $x, y$ : $100 \mu \mathrm{m} . \boldsymbol{B}$, In contrast, at $6 \mathrm{~h}$ after $\mathrm{SCl}$, many leukocytes are present within the posterior vein (shown) and postcapillary venules in isotype-treated animals (top) compared with anti-Ly6G/Gr-1-treated animals (bottom). Scale bar, $x, y$ : $100 \mu \mathrm{m}$. C, Quantification of rolling flux (cells/min/100 $\mu \mathrm{m}$ ) (top) and firm adherence (cells stationary for $30 \mathrm{~s}$ or longer $/ 100 \mu \mathrm{m}^{2}$ ) (bottom) reveals that anti-Ly6G/Gr-1 treatment significantly $(p<0.001$, ANOVA) reduces both neutrophil rolling and firm adhesion at both 6 and $12 \mathrm{~h}$ after injury. $D$, A higher magnification representative image of the exposed spinal cord vasculature from video captured at $6 \mathrm{~h}$ after $\mathrm{SCl}$ using spinning disk confocal microscopy. Note that the majority of rhodamine 6G-positive leukocytes (red channel) used for quantification in Care indeed neutrophils (Gr-1 positive, blue channel), Scale bar: $20 \mu \mathrm{m}$. At $48 \mathrm{~h}$ after injury, anti-Ly6G/Gr-1 treatment ( $\boldsymbol{E}$, right) markedly reduced the number of neutrophils (Gr-1, blue channel) compared with isotype controls ( $\boldsymbol{E}$, middle) interacting with the endothelium (PECAM-1, green channel). In contrast, few neutrophils were present in uninjured blood vessels $(\boldsymbol{E}$, left). Data are represented as mean \pm SEM per group, ANOVA with Holm-Sidak multiple comparison test. ${ }^{* *} p<0.01,{ }^{*} p<0.05,{ }^{*} p<0.05$ sham versus all other groups; ${ }^{\dagger} p<0.05,15$ min after $\mathrm{SCl}$ versus 6 and $12 \mathrm{~h}$ after $\mathrm{SCl}$ isotype controls, $n=3-5$ per group.

spinal cord using flow cytometry as previously described (Stirling and Yong, 2008). Previous work showed that spinal cord tissue from sham and naive controls has few neutrophils present, whereas SCI elicits a robust neutrophil infiltration that peaks at 12-24 h after injury (Stirling and Yong, 2008). In agreement with our previous study, few neutrophils were present within the spinal cord of sham-injured controls (data not shown), whereas SCI induced a robust accumulation of neutrophils within the injured tissue (Fig. $3 A, B$ ). Importantly the anti-Ly6G/Gr-1 strategy induced a pronounced and significant $(p<0.001)$ reduction in neutrophil (CD45 ${ }^{\text {high }}: \mathrm{CD} 11 \mathrm{~b}^{\text {high }}: \mathrm{Gr}-1^{\text {high }}$ ) infiltration at both 24 and $48 \mathrm{~h}$ after SCI (Fig. 3C). Our previous work (Stirling and Yong, 2008) had shown that the CD $45^{\text {high }}$ : CD11b ${ }^{\text {high }}:$ Gr- $1^{\text {high }}$ populations were not monocytoid cells by virtue of the absence of F4/80 and CD107b.

To rule out the possibility that antiLy6G/Gr-1 therapy masked the Gr-1 epitope and thus reduced the amount of available Gr-1 for subsequent staining, we also assessed CD11b levels. As shown in Figure $3 A, B, C D 45^{\text {high }}:$ CD $11 b^{\text {high }}$ neutrophils were markedly reduced compared with resident microglia (CD45 ${ }^{\text {low }}$ : $\mathrm{CDllb}^{+}$) in anti-Ly6G/Gr-1-treated mice. As revealed in supplemental Figure 1 (available at www.jneurosci.org as supplemental material), the majority of neutrophils (GFP-positive cells from Lyse-GFP mice) were parenchymal rather than adherent within blood vessels by $24 \mathrm{~h}$ after injury, with morphology consistent with migration through tissue.

To further confirm the reduction of neutrophils within the injured spinal cord, immunohistochemistry was used to assess neutrophil accumulation within the lesion site. Lyse-GFP mice confirmed the reduction of invading neutrophils within the injured spinal cord at $24 \mathrm{~h}$ after injury in anti-Ly6G/Gr-1-treated mice and revealed close association of microglia/macrophages (Iba-1 positive) with GFP-positive neutrophils (Fig. 3D-G; supplemental Fig. 1, available at www.jneurosci.org as supplemental material). At $48 \mathrm{~h}$ after injury, neutrophils (labeled by the marker $7 / 4$ ) were clearly reduced in anti-Ly6G/Gr-1treated mice compared with isotype controls (Fig. $3 H-M$ ).

\section{Anti-Ly6G/Gr-1 antibody treatment} alters cytokine and growth factor levels important for wound healing after SCI without altering microglia/macrophage density

The capacity to profoundly reduce neutrophil numbers in the injured spinal cord using anti-Ly6G/Gr-1 treatment provides the means to determine the pathological roles of neutrophil accumulation. We first used the RayBio Mouse G Series Inflammation Array I protein array that measures 40 inflammatory molecules within the spinal cord at $48 \mathrm{~h}$ after injury; at this time point, there were visible differences in lesion size and spared white matter between the groups (data not shown). We found pronounced and significant changes in cytokine and chemokine protein levels in anti-Ly6G/Gr-1treated mice versus isotype controls (Fig. 4). Noticeably, macrophage inflammatory protein $1-\gamma$ (MIP1 $\gamma /$ CCL9) levels, which increased $\sim 40$-fold after SCI, were elevated further in neutrophil-depleted mice (Fig. $4 A$ ). There were also signifi- 
A

24 hours after SCI

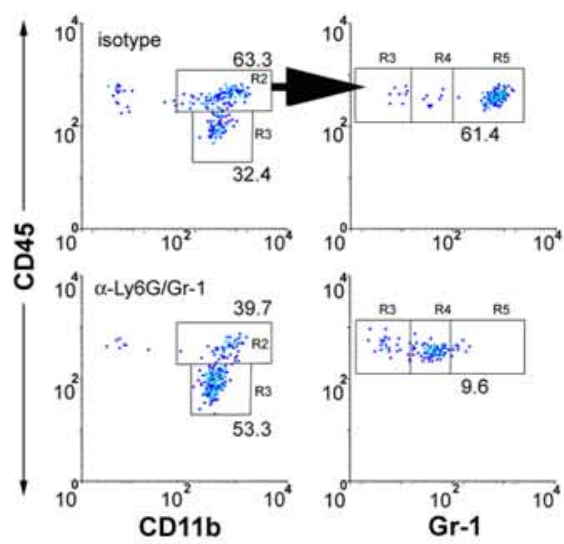

B

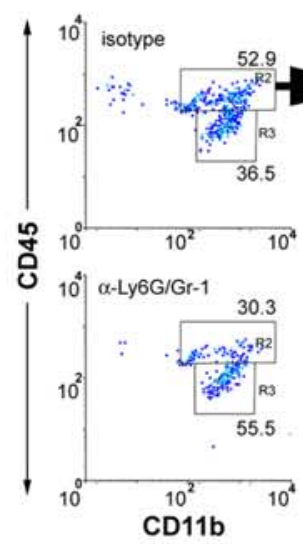

C

SCl
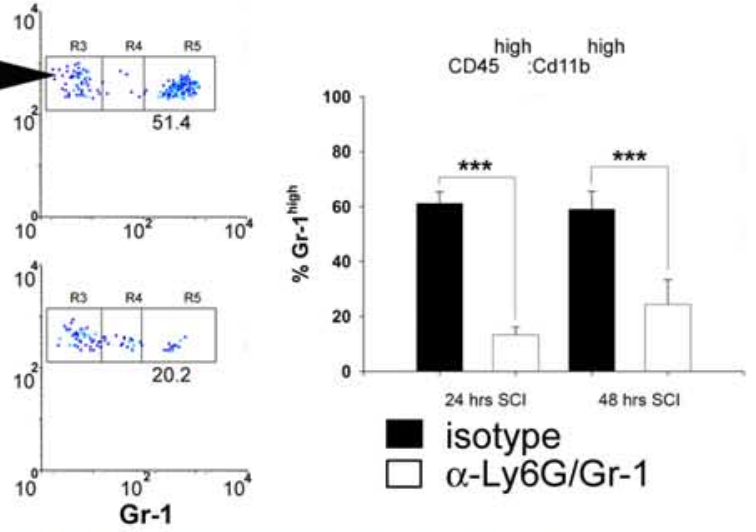

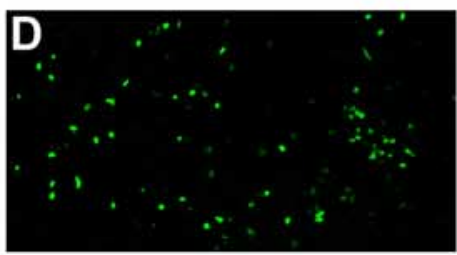

$\mathbf{E}$
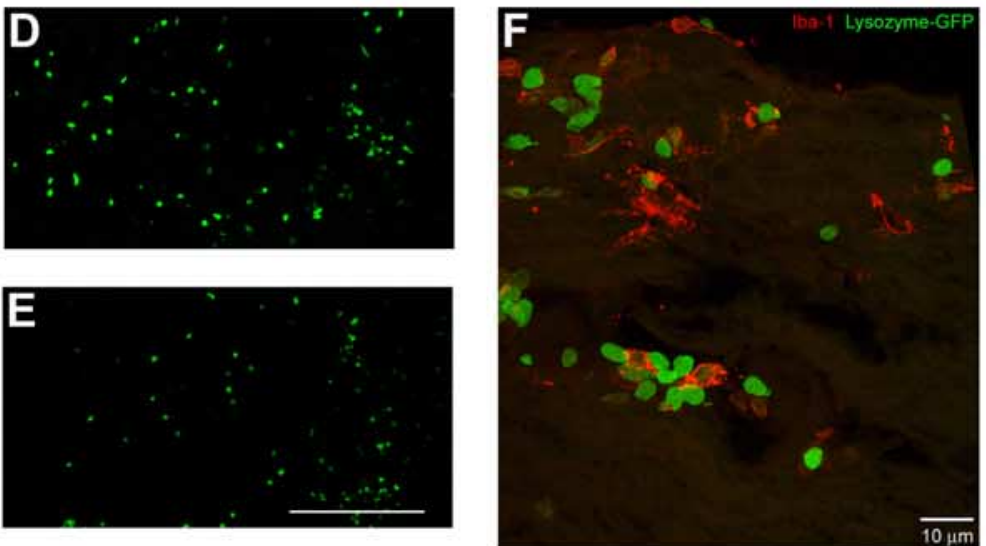

$\overline{10 \mu \mathrm{m}}$
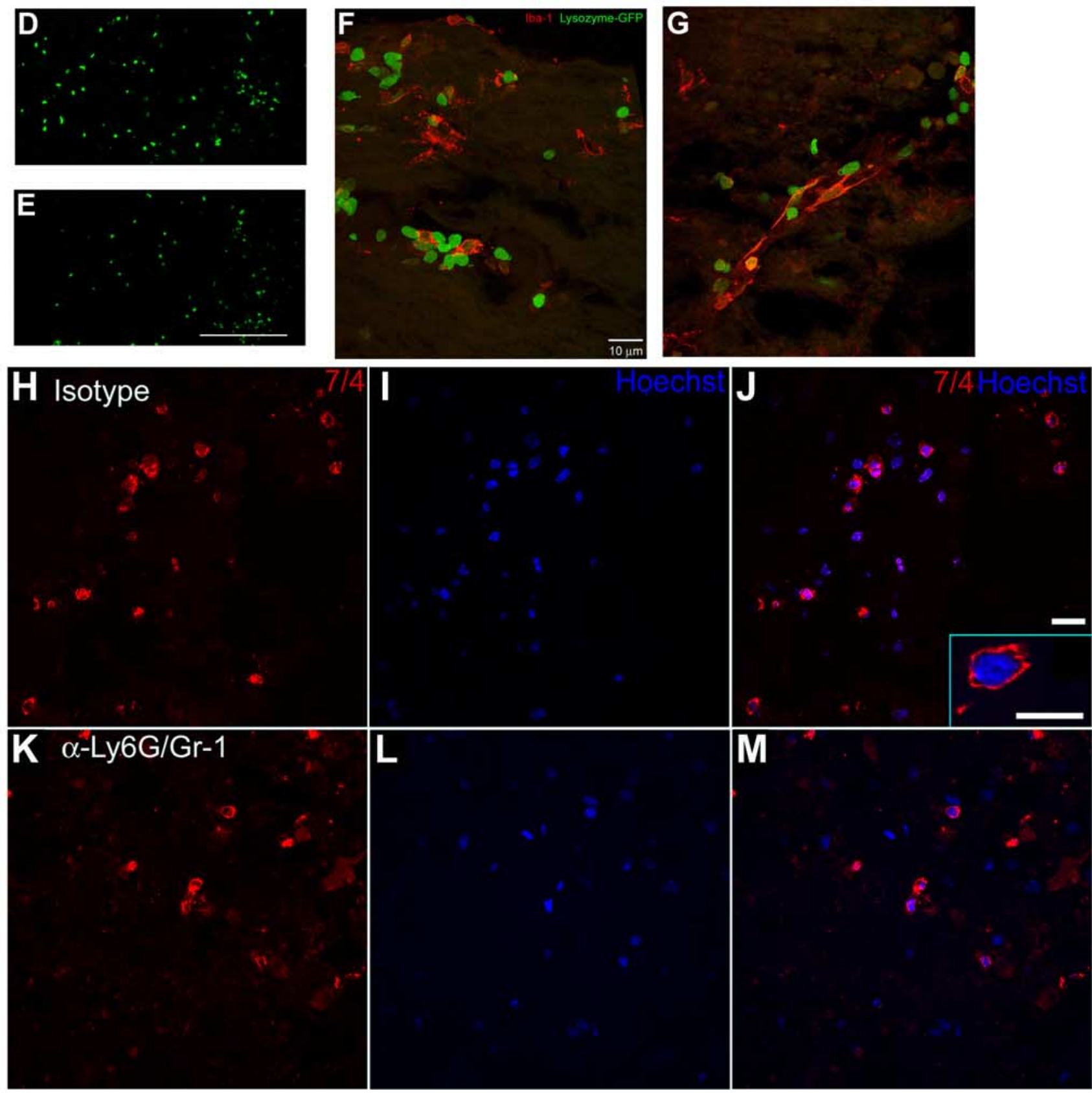

Figure 3. $A-M, A n t i-L y 6 G / G r-1$ treatment reduces neutrophil accumulation within the injured spinal cord. Representative density plots of injured spinal cord cells at $24 \mathrm{~h}(\boldsymbol{A})$ and $48 \mathrm{~h}(\boldsymbol{B})$ after injury. Microglia (CD45 ${ }^{\text {low. }}$ :CDllb ${ }^{+}$, boxed region R3), and blood-derived myeloid cells (CD45 high.:CDllb ${ }^{+}$, boxed region R2), are differentiated using flow cytometry. Further separation of neutrophils (CD45 ${ }^{\text {high }}$ :CDllb $\left.{ }^{\text {high }} \mathrm{Gr}^{\text {h }}{ }^{\text {high }}\right)$ is shown in R5. The percentage of each population is indicated. As shown in $\boldsymbol{A}$ and $\boldsymbol{B}$, neutrophils (R5) are clearly reduced at (Figure legend continues.) 


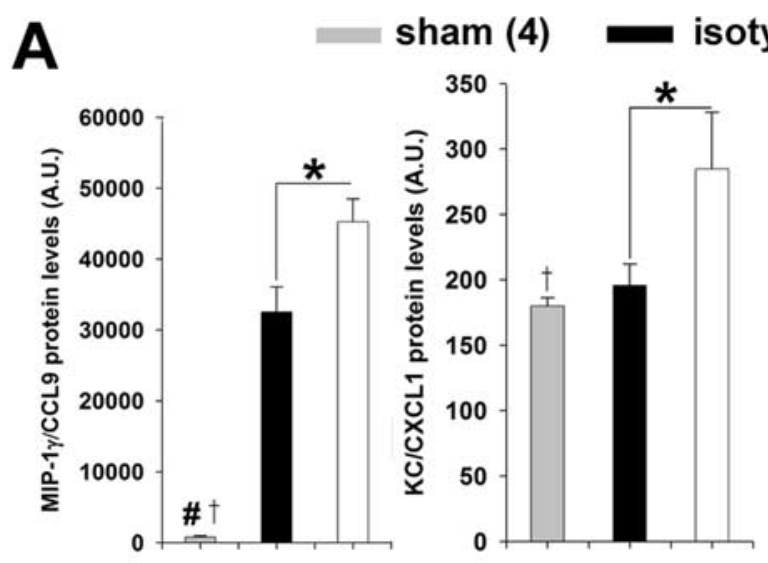

s-Ly6G/Gr-1 (4)

B

48 hours
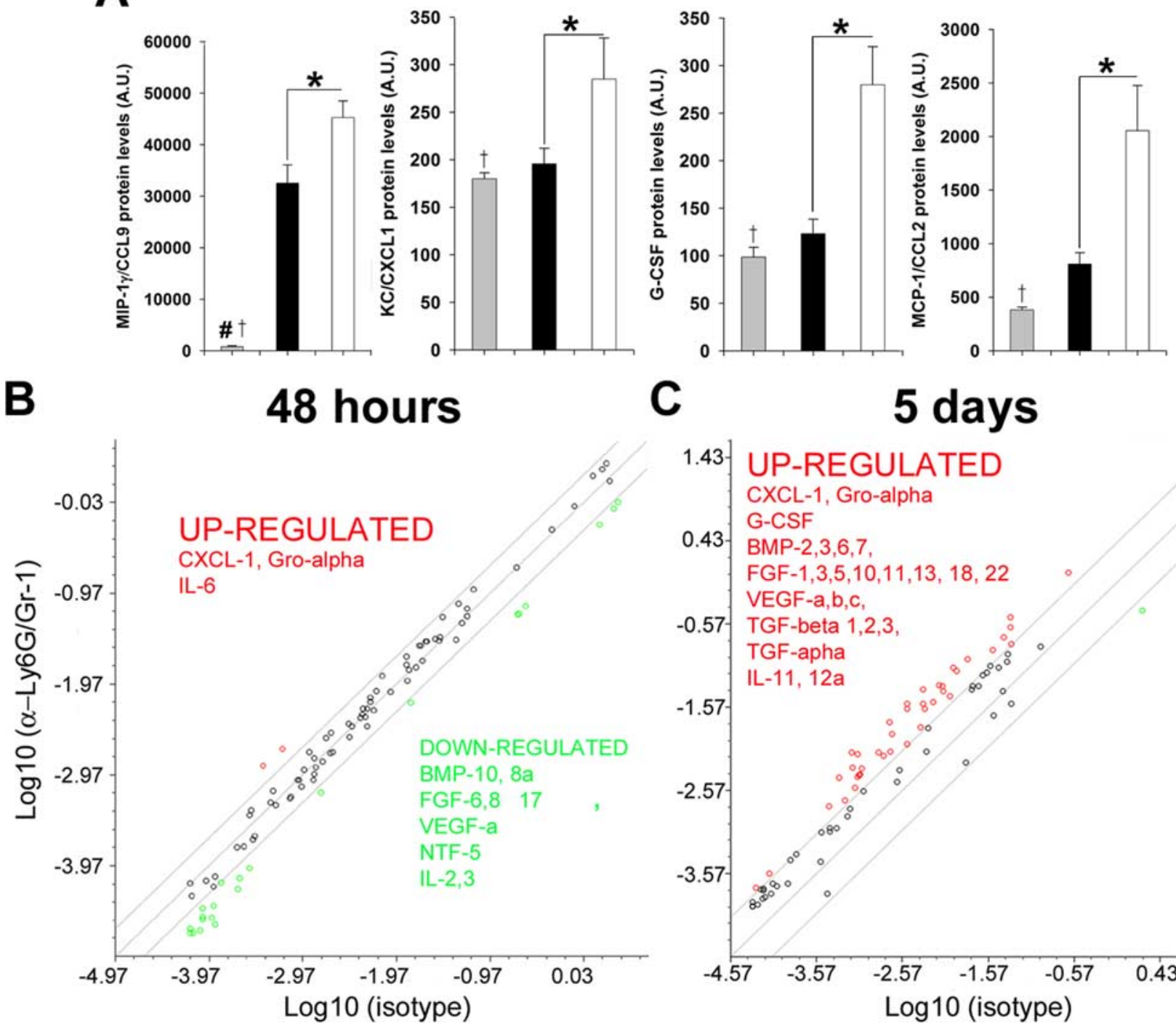

C

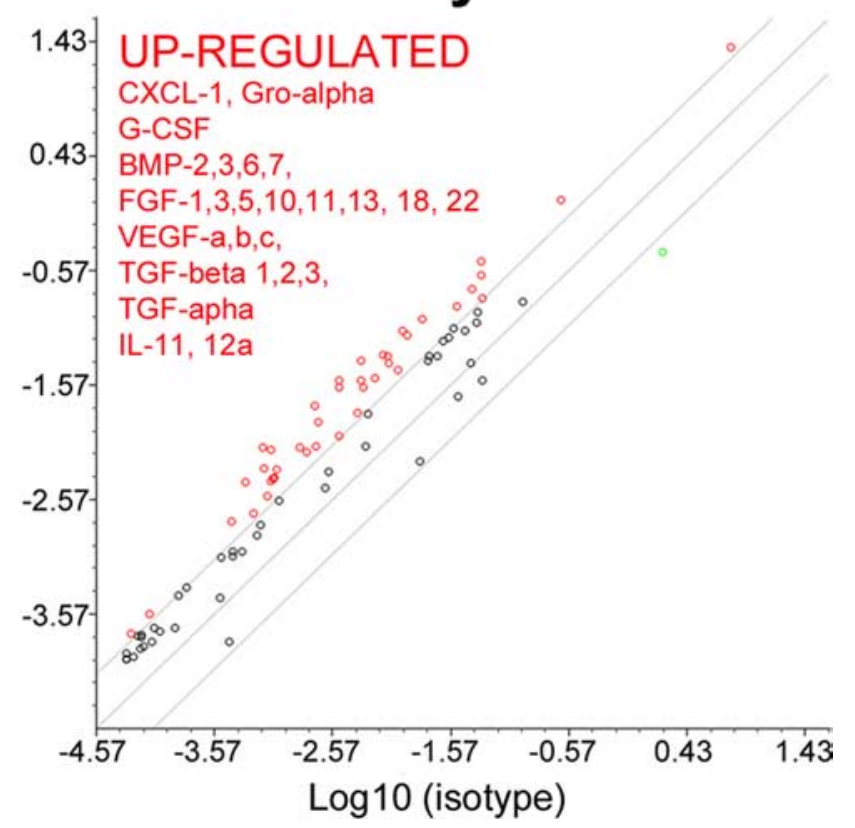

Figure 4. Anti-Ly6G/Gr-1 treatment alters cytokine and growth factor levels within the injured spinal cord at $48 \mathrm{~h}$ and $5 \mathrm{~d}$ after SCl. A, Quantification of inflammatory protein levels isolated from the injured spinal cord at $48 \mathrm{~h}$ after injury reveals a significant increase in MIP-1 $\gamma / C C L 9, \mathrm{KC} / \mathrm{CXCL1}$, G-CSF, and MCP-1/CCL2 protein levels in neutrophil-depleted animals (white bars) versus isotype (black bars) or sham controls (gray bars). Data are represented as mean protein levels [arbitrary units (A.U.)] \pm SEM, $n=4$ per group. ANOVA, Holm-Sidak method. ${ }^{*} p<0.05$, isotype versus $\alpha$-Ly6G/Gr-1-treated animals. ${ }^{*} p<0.05$, sham versus isotype. ${ }^{\dagger} p<0.05$ sham versus $\alpha$-Ly6G/Gr-1-treated animals. Analysis of growth factor mRNA levels isolated from the injured spinal cord at $48 \mathrm{~h}(\boldsymbol{B})$ and $5 \mathrm{~d}(\boldsymbol{C})$ after SCl using quantitative RT-PCR arrays. Plots of growth factor gene expression with a threefold change or higher are displayed, with $\alpha$-Ly6G/Gr-1-treated ( $y$-axis) and isotype controls ( $x$-axis) in $\boldsymbol{B}$ and $\boldsymbol{C}$. Upregulated genes are shown in red, whereas downregulated genes are shown in green. MCP-1/CCL2 monocyte chemoattractant protein-1, KC/CXCL1 keratinocyte derived chemokine, G-CSF granulocyte colony stimulating factor, MIP-1 $\gamma / C C L 9$, macrophage inflammatory protein-1 gamma, Bone morphogenic protein (BMP), fibroblast growth factor (FGF), Interleukin (IL), neutrotrophic factor (NTF), Transforming Growth factor (TGF), vasculature endothelial growth factor (VEGF).

cantly higher levels in the anti-Ly6G/Gr-1 treatment group compared with isotype controls of the neutrophil chemoattractant keratinocyte-derived chemokine KC/Gro- $\alpha /$ CXCL1, the neutrophil myelopoietic granulocyte-colony stimulating factor (G-CSF), and the monocyte chemoattractant protein-1
(MCP1/CCL2) (Fig. 4A). In contrast, anti-Ly6G/Gr-1 treatment did not cause a significant change in cytokine protein levels proposed to play a role in SCI pathology or repair including GM-CSF, M-CSF, interleukin (IL)-1 $\beta$, IL-10, and tumor necrosis factor $\alpha$ (data not shown).

(Figure legend continued.) 24 and $48 \mathrm{~h}$ after SCl in anti-Ly6G/Gr-1-treated mice (bottom) versus isotype controls (top). C, Quantification of blood-derived myeloid cells entering the spinal cord reveals that the percentage of neutrophils is significantly $(p<0.001$ ) reduced at both 24 and $48 \mathrm{~h}$ after injury in neutrophil-depleted mice (white bars) compared with isotype control animals (black bars). Data are represented as mean percentage $\pm \mathrm{SEM}, n=4-5$ per group, ANOVA. ${ }^{* * *} p<0.001$. Lysozyme-GFP mice reveal that fewer myeloid cells (green) enter the injured spinal cord in neutrophil-depleted mice $(\boldsymbol{E})$ compared with isotype controls $(\boldsymbol{D})$ at $24 \mathrm{~h}$ after SCI. Scale bar, $200 \mu \mathrm{m}$. Higher magnification images show several myeloid cells (GFP, green) in close contact to Iba-1-positive (red) microglia/macrophages $(\boldsymbol{F})$ in isotype animals, whereas fewer myeloid cells are present in neutrophil-depleted mice $(\boldsymbol{G})$. Scale bar, $10 \mu \mathrm{m}$. $\boldsymbol{H}-\boldsymbol{M}, \mathbf{G r e a t e r}$ numbers of neutrophils are evident within the injured spinal cord at $48 \mathrm{~h}$ after $\mathrm{SCl}$ in isotype control animals using the neutrophil-specific marker $7 / 4(\mathrm{red}, \mathrm{H})$ compared with neutrophil-depleted mice $(\boldsymbol{K})$. Merged images of neutrophils in neutrophil-depleted injured spinal cord lesions $(M)$ and isotype animals $(J)$ are shown. The nuclear stain Hoechst (blue) reveals the polymorphic nucleus characteristic of neutrophils (I, $L$; J, inset). Scale bar: (J) for $\boldsymbol{H}-M$ : $20 \mu \mathrm{m}$; for $\boldsymbol{J}$, inset: $10 \mu \mathrm{m}$. 
The increases in chemokine (CCL9, CXCL1, and CCL2) and cytokine (G-CSF) levels within the CNS of anti-Ly6G/Gr-1treated mice may represent attempts at compensation to recruit more inflammatory cells into the injured spinal cord. To test this, and because CCL9 and CCL2 are monocytoid chemoattractants, we assessed microglia/macrophage density within the lesion site at $5 \mathrm{~d}$ after injury using the microglia/macrophage marker Iba-1. As shown in supplemental Figure 2 (available at www.jneurosci.org as supplemental material), there was not a significant $(p=0.866)$ difference in Iba-1 density between the groups. Thus, anti-Ly6G/ Gr-1 treatment did not lead to any obvious compensatory increase in macrophage/ microglia numbers within the spinal cord, despite an elevation of chemokine levels.

The changes in endogenous wound healing and tissue remodeling between the treatment groups were then assessed. Total RNA from injured spinal cord tissue was used for quantitative RT-PCR superarrays to assess levels of transcripts encoding growth factors and inflammatory molecules. As shown in Figure $4 B$, several growth factors involved in wound healing and tissue remodeling were suppressed at $48 \mathrm{~h}$ but, interestingly, augmented at $5 \mathrm{~d}$ in anti-Ly6G/Gr1-treated mice compared with isotype controls. At $48 \mathrm{~h}$, genes significantly downregulated in anti-Ly6G/Gr-1treated mice included bone morphogenic proteins (BMPs), fibroblast growth factors (FGFs), vascular endothelial growth factors (VEGFs), neurotrophic factors (NTFs), and IL-2 and -3; conversely, there was upregulation of IL- 6 and the neutrophil chemoattractant $\mathrm{KC} / \mathrm{Gro}-\alpha / \mathrm{CXCL} 1$. In contrast, at $5 \mathrm{~d}$ after injury, compared with isotype controls, anti-Ly6G/Gr-1-treated animals expressed high levels (Fig. 4C) of BMPs, FGFs, VEGFs, and the TGF family members. These results suggest that neutrophils may be important in signaling early wound healing events after SCI.
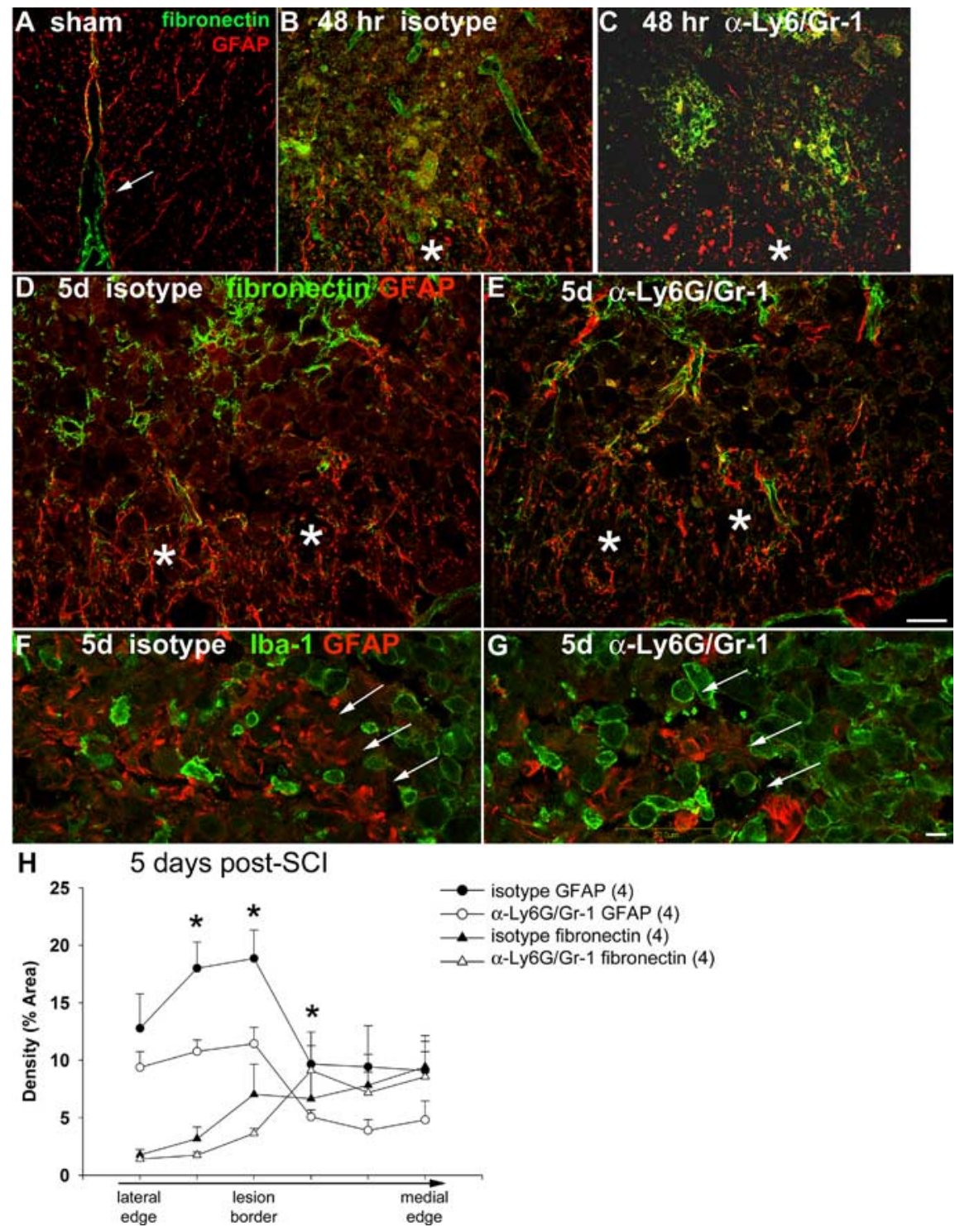

Figure 5. Anti-Ly6G/Gr-1 treatment dampens the evolution of astrocyte reactivity after SCI. $\boldsymbol{A}$, In sham-injured controls fibronectin (green) is mainly localized to blood vessels and connective tissue as expected. The medial aspect of the ventral column is shown (arrow). Low levels of GFAP (red) delineate astrocytes. In contrast, at $48 \mathrm{~h}(\boldsymbol{B}, \boldsymbol{C})$ and $5 \mathrm{~d}(\boldsymbol{D}, \boldsymbol{E})$ after injury, the evolution of the glial scar is apparent and dense, as revealed using GFAP immunoreactivity in isotype animals $(\boldsymbol{B}, \boldsymbol{D})$, but much less reactivity is seen in anti-Ly6G/Gr-1 neutrophil-depleted mice $(\boldsymbol{C}, \boldsymbol{E})$. Asterisks mark the area of maximum GFAP density. Fibronectin staining is indistinguishable between the two groups. Scale bar, $25 \mu \mathrm{m}$. Higher magnification images show Iba-1 (green)-positive microglia/macrophages invading past the less densely formed glial scar (GFAP, red) in neutrophil-depleted mice (G) compared with isotype controls $(\boldsymbol{F})$. Scale bar, $10 \mu \mathrm{m}$. $\boldsymbol{H}$, Quantification of the glial scar at $5 \mathrm{~d}$ after injury reveal a significant increase in GFAP signal (percentage area) in isotype (filled circles)- versus anti-Ly6G/Gr-1 (open circles)-treated animals. There was not a significant difference in fibronectin density between the groups. Data are represented as mean percentage $\pm S E M, n=4$ four per group, Mann-Whitney rank sum test, ${ }^{*} p<0.05$.

\section{Anti-Ly6G/Gr-1 antibody treatment alters glial scar formation after SCI}

The clue that neutrophils recruited after SCI could be important for wound healing events led us to examine the formation of the glial scar after injury. An early step of this response is the activation of astrocytes that is necessary to reform a damaged blood-brain barrier, to detoxify toxic substances, and for general wound repair (Faulkner et al., 2004; Herrmann et al., 2008). There is also remodeling of the extracellular matrix manifested by alterations of molecules such as fibronectin by astrocytes (Tom et al., 2004). Thus, we assessed immunoreactivity for fibronectin and GFAP, representative of the evolu- tion of astrocyte reactivity, in the lesion site from anti-Ly6G/ Gr-1- or isotype-treated animals at $48 \mathrm{~h}$ and $5 \mathrm{~d}$ after SCI. We measured these signals from the lateral edge toward the lesion center to evaluate whether there was a difference in gradient formation of the glial scar between groups. As shown in Figure 5 , SCI induced a rise in fibronectin and GFAP levels at $48 \mathrm{~h}$, which increased in intensity at $5 \mathrm{~d}$ after injury. Strikingly, neutrophil depletion attenuated the injury-induced rise of GFAP levels $(p<0.05)$ while not affecting fibronectin staining. In addition, Iba-1-positive microglia/macrophages were seen expanding further into spared white matter in neutrophil-depleted animals compared with controls (Fig. 5). 

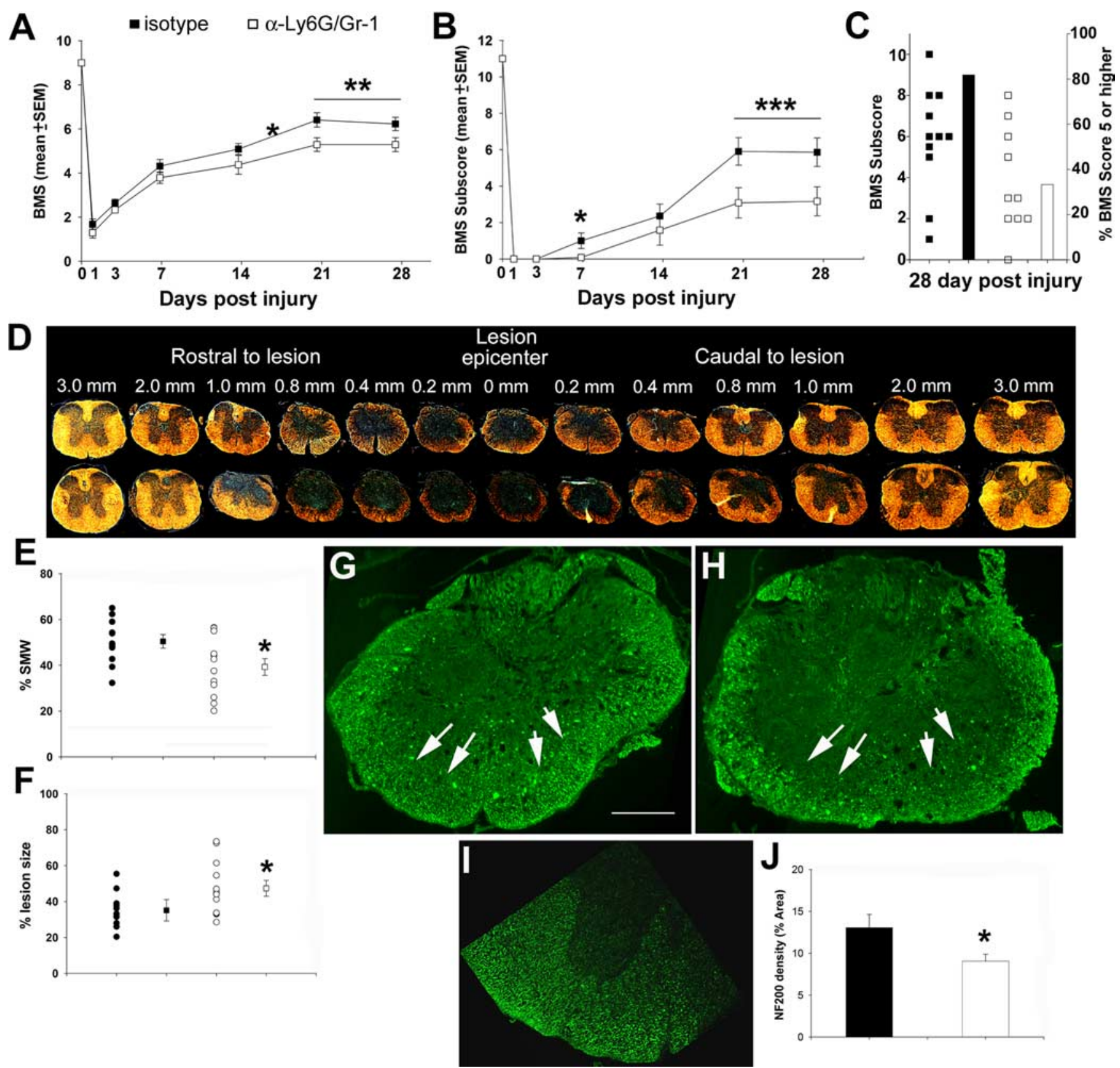

Figure 6. $\quad A, B, A n t i-L y 6 G / G r-1$ treatment worsens neurological outcome after SCI. Neutrophil depletion (open squares) worsened functional outcome as revealed by BMS ( $A$ ) and BMS subscores (B) compared with isotype controls (filled squares). C, Individual mice BMS subscore values are shown at $28 \mathrm{~d}$ after injury ( $y$-axis, left) (filled squares, isotype; open squares, anti-Ly6G/Gr-1). Percentage of animals to reach a BMS subscore of 5 and higher at $28 \mathrm{~d}$ after injury is also shown ( $y$-axis, right) (filled bar, isotype; open bar, anti-Ly6G/Gr-1). Data are represented as mean \pm SEM, $n=11-12$ per group; RM-ANOVA, Holm-Sidak method. ${ }^{*} p<0.05 .{ }^{* *} p<0.01,{ }^{* * *} p<0.001$. D, Representative eriochrome cyanine- and neutral red-stained serial sections throughout the lesion site from isotype-treated (top panels) and anti-Ly6G/Gr-1-treated (bottom panels) animals. Note that at the lesion epicenter, more spared white matter (SWM, yellow) and smaller lesions are evident in the isotype-treated animal. Quantification of SWM $(\boldsymbol{E})$ and lesion size $(\boldsymbol{F})$ reveals significantly less SWM and greater lesion size in neutrophil-depleted (open circles, individual animals; open square, mean) versus isotype control animals (filled circles; filled square, mean). Data are represented as mean percentage \pm SEM, $n=11-12$ per group, Mann-Whitney Rank Sum test, ${ }^{*} p<$ 0.05. G-I, NF200 staining of the lesion epicenter from an isotype $(\boldsymbol{G})$, a neutrophil-depleted $(\boldsymbol{H})$, or a sham-control animal $(\boldsymbol{I})$ is shown [(scale bar: $(\mathbf{G}-\boldsymbol{I})$, $500 \mu \mathrm{m}$. Less NF200 staining is evident in neutrophil-depleted versus isotype controls (arrows in $\boldsymbol{G}$ and $\boldsymbol{H}$ ). Quantification of axonal density (NF200 percentage of area occupied) reveals a significant decrease in NF200 density in neutrophil depleted animals (open bars) versus isotype-treated animals (filled bars). Data are represented as mean \pm SEM, $n=11-12$ per group, Mann-Whitney Rank Sum test, ${ }^{*} p<0.05$.

These data suggest a reduced wound healing and scar formation response in neutrophil-depleted animals.

Anti-Ly6G/Gr-1 antibody treatment worsens behavioral and histological outcomes after SCI

The altered growth factor and GFAP responses resulting from anti-Ly6G/Gr-1 treatment suggest that there could be longterm behavioral and histological consequences. To address the former, we used the BMS, an established scoring system to assess locomotor recovery after SCI in mice (Basso et al., 2006). We found that anti-Ly6G/Gr-1 treatment significantly $(p<0.001$, repeated-measures ANOVA) worsened functional recovery after SCI (Fig. 6A). In this regard, anti-Ly6G/Gr-1treated mice had lower BMS scores, beginning early after injury, that reached significance at day $14(4.4 \pm 0.4$, mean \pm SEM) versus isotype control animals $(5.1 \pm 0.3, p<0.05$; 
Holm-Sidak method). Significant differences in BMS scores between the groups were maintained to $28 \mathrm{~d}$ after injury (neutrophil-depleted, $5.3 \pm 0.3$ vs isotype controls, $6.2 \pm 0.3$, $p<0.01$ ) (Fig. 6A). That this difference was not the result of our isotype treatment was affirmed by comparing these animals with saline-treated mice after SCI, in which there was no significant difference between the two groups (data not shown).

We next used BMS subscores that assess finer aspects of hindlimb movement independent of whether the animal reaches forelimb-hindlimb coordination. These subscores reveal that anti-Ly6G/Gr-1-treated mice had significantly inferior functional outcome beginning at $7 \mathrm{~d}$ and at 21 and $28 \mathrm{~d}$ after injury, compared with isotype controls (Fig. $6 \mathrm{~B}$ ). Indeed, only $33 \%$ of anti-Ly6G/Gr-1-treated mice reached a BMS subscore of 5 and higher by $28 \mathrm{~d}$ after SCI, compared with $82 \%$ in the isotype-treated animals (Fig. 6C). Thus, anti-Ly6G/Gr-1 treatment worsens functional outcome after SCI.

At the end of the behavioral observation period at day 28 , mice were killed to determine whether anti-Ly6G/Gr-1 treatment altered the amount of spared white matter and lesion size after SCI. Serial sections throughout the lesion site of representative isotype and anti-Ly6G/Gr-1-treated animals $28 \mathrm{~d}$ after injury are shown in Figure $6 D$. Eriochrome cyanine staining for myelin and neutral red stain to assess lesion size revealed less spared white matter from anti-Ly6G/Gr-1 treatment $(40.9 \pm 3.8$, mean percentage \pm SEM $)$ versus isotype control (51.9 \pm 2.4\%, $p<0.05$ ) (Fig. 6D,E). Conversely, anti-Ly6G/Gr-1-treated mice had significantly larger lesions $(47.4 \pm 4.4$ vs $36.0 \pm 2.8$, isotype controls) (Fig. $6 F$ ).

We next addressed whether anti-Ly6G/Gr-1 treatment affected axonal sparing after SCI by evaluating NF200-stained sections from the lesion site. At $28 \mathrm{~d}$ after contusion injury, NF200 was reduced to a small ring of spared tissue in the lateral and ventral columns in isotype control animals (Fig. $6 G)$. In contrast, less NF200 staining was evident in anti-Ly6G/ Gr-1-treated mice (Fig. 6H). Quantification of these results revealed reduced axonal sparing in anti-Ly6G/Gr-1-treated mice $(9.1 \pm 0.8$, mean percentage \pm SEM) compared with isotype-treated animals $(13.1 \pm 1.6)$ (Fig. 6J). Collectively, anti-Ly6G/Gr-1 treatment resulted in decreased preservation of white matter and axons along with a poorer functional outcome after SCI.

\section{Discussion}

We have used powerful imaging techniques in vivo to allow, for the first time, the visualization of a robust recruitment of neutrophils to spinal cord microvasculature within the lesion site, where they begin to tether and roll onto endothelial cells as early as $15 \mathrm{~min}$ after SCI, before their entry into the injured spinal cord. Firm adhesion to endothelial cells, a prerequisite for subsequent neutrophil diapedesis, was evident at $6-12 \mathrm{~h}$ and continued to $48 \mathrm{~h}$ after injury. Our study provides not only visualization in real time but also the quantitation of leukocyte dynamics in blood vessels around SCI lesion sites, which should be helpful in future studies that target particular immune subsets in the circulation to modulate the extent of secondary injury after SCI.

Given the extensive and early influx of neutrophils, we used the anti-Ly6G/Gr-1 antibody and found that it diminished circulating neutrophils after SCI (Fig. 1A). The anti-Ly6G/ Gr-1 antibody also reduced neutrophil trafficking within the posterior spinal vein and venules of the lesion site (Fig. $2 E$ ), and it markedly attenuated neutrophil invasion into the injured spinal cord (Fig. 3C).

We are cognizant that the RB6-8C5 clone of anti-Ly6G/ Gr-1 antibody not only binds the Ly6G/Gr-1 antigen on mature neutrophils (Hestdal et al., 1991; Fleming et al., 1993), but has also been reported to recognize Ly6C, which is found on a subset of monocytoid cells and lymphocytes (Fleming et al., 1993; Daley et al., 2008). Thus, in addition to the depletion of neutrophils in blood and the injured spinal cord, it is possible that the anti-Ly6G/Gr-1 antibody might have depleted monocytoid cells and lymphocytes. Others, however, have not found the RB6-8C5 clone to cross-react with Ly6G (Nagendra and Schlueter, 2004). In our study, we were unable to document obvious changes to monocyte or lymphocyte numbers in blood after SCI as a result of antibody treatment (Fig. $1 B, C$ ), and we did not observe changes to microglia/macrophage density within the injured spinal cord. Thus, our collective results suggest that the anti-Ly6G/Gr-1 antibody reduced neutrophils while leaving other cell types intact, although we cannot rule out the possibility that a small but important subset of monocytoid or lymphoid cells was altered in number or activity by treatment. Recently, Daley et al. (2008) reported that another Ly6G-specific clone, 1A8, decreased blood neutrophils but not monocytes. It would be of interest in future studies to address whether there are differential outcomes after SCI to the RB6$8 \mathrm{C} 5$ and $1 \mathrm{~A} 8$ clones.

The treatment of mice with anti-Ly6G/Gr-1, with the resultant profound loss of neutrophils, surprisingly worsened neurological recovery, which was associated with increased lesion area, decreased spared white matter, an altered cytokine and growth factor milieu, and diminished wound healing as indicated by reduced astrocyte reactivity. Together, these results suggest an important role for neutrophil recruitment in implementing wound healing and protective events that limit secondary tissue loss after SCI.

The possibility that neutrophils are beneficial after SCI is unexpected because the massive influx of neutrophils into the CNS has been anticipated to be detrimental; this is because the products of neutrophils, including free radicals, proteases, and cytokines, can injure tissue. However, inflammatory responses are complex, and benefits of inflammation to the CNS are increasingly being appreciated (Schwartz and Yoles, 2006; Popovich and Longbrake, 2008). Indeed, in support of our finding for a beneficial role for neutrophil recruitment, others have documented more oxidative stress within the injured cord in the absence of neutrophils (de Castro et al., 2004). Furthermore, stimulated neutrophils were reported to release IL-1 receptor antagonist (Schröder et al., 2006), a potential neuroprotective agent, after SCI (Nesic et al., 2001).

A probable mechanism for the benefit of neutrophils in SCI is their involvement in the wound healing response. Although scar formation provides a barrier for axonal regeneration, several recent studies found that reduced astrocyte activity and astrogliosis after SCI worsened functional outcome (Faulkner et al., 2004; Herrmann et al., 2008; Renault-Mihara et al., 2008). Reactive astrocytes help seal off and isolate the necrotic core of the lesion site, help reestablish the integrity of the blood-brain barrier, and detoxify molecules such as free radicals and glutamate (Sofroniew, 2005). Thus, we propose that neutrophils help to promote the astrocyte response after SCI, a novel hypothesis, and further studies will be needed to elucidate the precise signals involved.

An orchestrated growth factor and chemokine/cytokine 
milieu that guides both infiltrating and resident CNS cells to promote endogenous repair is likely of importance in wound healing after SCI. In the present study many of the chemokines and colony-stimulating factors important for granulopoiesis and neutrophil recruitment were increased in neutrophildepleted animals at $48 \mathrm{~h}$ (Fig. $4 \mathrm{~A}$ ), suggesting a compensatory mechanism to help recruit more neutrophils into the neutropenic spinal cord. However, because the anti-Ly6G/Gr-1 treatment depletes bone marrow reserves of neutrophils (our unpublished data) (Fleming et al., 1993), few cells were able to enter the spinal cord. Notably, the growth factor milieu within the injured spinal cord was downregulated at $48 \mathrm{~h}$ after injury in the neutrophil-depleted animals. Many of these growth factors, including FGFs, VEGFs, and BMPs, may be important for promoting wound healing; thus, their deficiency may underlie the reduced astrocyte reactivity seen in neutrophil-depleted animals. In contrast to the $48 \mathrm{~h}$ time point, growth factors were clearly augmented in neutrophil-depleted animals at $5 \mathrm{~d}$ after injury. The significance of the higher levels of growth factors at $5 \mathrm{~d}$ in neutrophil-depleted mice is unknown. Elevated growth factor levels can sometimes be detrimental; for example, inhibitors to BMPs improve outcome after SCI (Matsuura et al., 2008). Similarly, FGFs increase levels of CHL1, an inhibitor of neurite outgrowth, which may hinder SCI repair (Jakovcevski et al., 2007). Clearly, the implications of altered growth factor responses in neutrophil-depleted mice are complex, and it is likely that the overall expression and spatial location of these factors drive the final outcome toward containment of the injury and endogenous repair.

In summary, although the spectrum of cells affected by anti-Ly6G/Gr-1 antibody treatment cannot be fully ascertained at this point, the significant depletion of neutrophils and worsened recovery suggest an unexpected beneficial role for neutrophil recruitment after SCI. These data caution against the use of nonspecific anti-inflammatory treatments for SCI until the precise roles of subsets of leukocytes including neutrophils are determined.

\section{References}

Bao F, Chen Y, Dekaban GA, Weaver LC (2004) Early anti-inflammatory treatment reduces lipid peroxidation and protein nitration after spinal cord injury in rats. J Neurochem 88:1335-1344.

Bartholdi D, Schwab ME (1995) Methylprednisolone inhibits early inflammatory processes but not ischemic cell death after experimental spinal cord lesion in the rat. Brain Res 672:177-186.

Bartholdi D, Schwab ME (1997) Expression of pro-inflammatory cytokine and chemokine mRNA upon experimental spinal cord injury in mouse: an in situ hybridization study. Eur J Neurosci 9:1422-1438.

Basso DM, Fisher LC, Anderson AJ, Jakeman LB, McTigue DM, Popovich PG (2006) Basso Mouse Scale for locomotion detects differences in recovery after spinal cord injury in five common mouse strains. J Neurotrauma 23:635-659.

Carlson SL, Parrish ME, Springer JE, Doty K, Dossett L (1998) Acute inflammatory response in spinal cord following impact injury. Exp Neurol 151:77-88

Conlan JW, North RJ (1994) Neutrophils are essential for early anti-Listeria defense in the liver, but not in the spleen or peritoneal cavity, as revealed by a granulocyte-depleting monoclonal antibody. J Exp Med 179:259-268.

Daley JM, Thomay AA, Connolly MD, Reichner JS, Albina JE (2008) Use of Ly6G-specific monoclonal antibody to deplete neutrophils in mice. J Leukoc Biol 83:64-70.

de Castro R Jr, Hughes MG, Xu GY, Clifton C, Calingasan NY, Gelman BB, McAdoo DJ (2004) Evidence that infiltrating neutrophils do not release reactive oxygen species in the site of spinal cord injury. Exp Neurol 190:414-424.

Donnelly DJ, Popovich PG (2008) Inflammation and its role in neuropro- tection, axonal regeneration and functional recovery after spinal cord injury. Exp Neurol 209:378-388.

Dumont RJ, Okonkwo DO, Verma S, Hurlbert RJ, Boulos PT, Ellegala DB, Dumont AS (2001) Acute spinal cord injury, part I: pathophysiologic mechanisms. Clin Neuropharmacol 24:254-264.

Dusart I, Schwab ME (1994) Secondary cell death and the inflammatory reaction after dorsal hemisection of the rat spinal cord. Eur J Neurosci 6:712-724

Faulkner JR, Herrmann JE, Woo MJ, Tansey KE, Doan NB, Sofroniew MV (2004) Reactive astrocytes protect tissue and preserve function after spinal cord injury. J Neurosci 24:2143-2155.

Faust N, Varas F, Kelly LM, Heck S, Graf T (2000) Insertion of enhanced green fluorescent protein into the lysozyme gene creates mice with green fluorescent granulocytes and macrophages. Blood 96:719-726.

Fleming JC, Norenberg MD, Ramsay DA, Dekaban GA, Marcillo AE, Saenz AD, Pasquale-Styles M, Dietrich WD, Weaver LC (2006) The cellular inflammatory response in human spinal cords after injury. Brain 129:3249-3269.

Fleming TJ, Fleming ML, Malek TR (1993) Selective expression of Ly-6G on myeloid lineage cells in mouse bone marrow. RB6-8C5 mAb to granulocyte-differentiation antigen (Gr-1) detects members of the Ly-6 family. J Immunol 151:2399-2408.

Forbes AD, Slimp JC, Winn RK, Verrier ED (1994) Inhibition of neutrophil adhesion does not prevent ischemic spinal cord injury. Ann Thorac Surg 58:1064-1068.

Gris D, Marsh DR, Oatway MA, Chen Y, Hamilton EF, Dekaban GA, Weaver LC (2004) Transient blockade of the CD11d/CD18 integrin reduces secondary damage after spinal cord injury, improving sensory, autonomic, and motor function. J Neurosci 24:4043-4051.

Herrmann JE, Imura T, Song B, Qi J, Ao Y, Nguyen TK, Korsak RA, Takeda K, Akira S, Sofroniew MV (2008) STAT3 is a critical regulator of astrogliosis and scar formation after spinal cord injury. J Neurosci 28:7231-7243.

Hestdal K, Ruscetti FW, Ihle JN, Jacobsen SE, Dubois CM, Kopp WC, Longo DL, Keller JR (1991) Characterization and regulation of RB6-8C5 antigen expression on murine bone marrow cells. J Immunol 147:22-28.

Holtz A, Nyström B, Gerdin B (1989) Spinal cord injury in rats: inability of nimodipine or anti-neutrophil serum to improve spinal cord blood flow or neurologic status. Acta Neurol Scand 79:460-467.

Jakovcevski I, Wu J, Karl N, Leshchyns'ka I, Sytnyk V, Chen J, Irintchev A, Schachner M (2007) Glial scar expression of CHL1, the close homolog of the adhesion molecule L1, limits recovery after spinal cord injury. J Neurosci 27:7222-7233.

Kerfoot SM, Norman MU, Lapointe BM, Bonder CS, Zbytnuik L, Kubes P (2006) Reevaluation of P-selectin and alpha 4 integrin as targets for the treatment of experimental autoimmune encephalomyelitis. J Immunol 176:6225-6234.

Kwon BK, Tetzlaff W, Grauer JN, Beiner J, Vaccaro AR (2004) Pathophysiology and pharmacologic treatment of acute spinal cord injury. Spine J 4:451-464.

Matsuura I, Taniguchi J, Hata K, Saeki N, Yamashita T (2008) BMP inhibition enhances axonal growth and functional recovery after spinal cord injury. J Neurochem 105:1471-1479.

Nagendra S, Schlueter AJ (2004) Absence of cross-reactivity between murine Ly-6C and Ly-6G. Cytometry A 58:195-200.

Nesic O, Xu GY, McAdoo D, High KW, Hulsebosch C, Perez-Pol R (2001) IL-1 receptor antagonist prevents apoptosis and caspase-3 activation after spinal cord injury. J Neurotrauma 18:947-956.

Norman MU, Hulliger S, Colarusso P, Kubes P (2008) Multichannel fluorescence spinning disk microscopy reveals early endogenous CD4 T cell recruitment in contact sensitivity via complement. J Immunol 180:510-521.

Pineau I, Lacroix S (2007) Proinflammatory cytokine synthesis in the injured mouse spinal cord: multiphasic expression pattern and identification of the cell types involved. J Comp Neurol 500:267-285.

Popovich PG, Longbrake EE (2008) Can the immune system be harnessed to repair the CNS? Nat Rev Neurosci 9:481-493.

Rabchevsky AG, Fugaccia I, Sullivan PG, Scheff SW (2001) Cyclosporin A treatment following spinal cord injury to the rat: behavioral effects and stereological assessment of tissue sparing. J Neurotrauma 18:513-522.

Rakhmilevich AL (1995) Neutrophils are essential for resolution of primary and secondary infection with Listeria monocytogenes. J Leukoc Biol $57: 827-831$. 
Renault-Mihara F, Okada S, Shibata S, Nakamura M, Toyama Y, Okano H (2008) Spinal cord injury: emerging beneficial role of reactive astrocytes' migration. Int J Biochem Cell Biol 40:1649-1653.

Rice T, Larsen J, Rivest S, Yong VW (2007) Characterization of the early neuroinflammation after spinal cord injury in mice. J Neuropathol Exp Neurol 66:184-195.

Schröder AK, von der Ohe M, Kolling U, Altstaedt J, Uciechowski P, Fleischer D, Dalhoff K, Ju X, Zenke M, Heussen N, Rink L (2006) Polymorphonuclear leucocytes selectively produce anti-inflammatory interleukin-1 receptor antagonist and chemokines, but fail to produce proinflammatory mediators. Immunology 119:317-327.

Schwartz M, Yoles E (2006) Immune-based therapy for spinal cord repair: autologous macrophages and beyond. J Neurotrauma 23:360-370.

Sofroniew MV (2005) Reactive astrocytes in neural repair and protection. Neuroscientist 11:400-407.
Sroga JM, Jones TB, Kigerl KA, McGaughy VM, Popovich PG (2003) Rats and mice exhibit distinct inflammatory reactions after spinal cord injury. J Comp Neurol 462:223-240.

Stirling DP, Yong VW (2008) Dynamics of the inflammatory response after murine spinal cord injury revealed by flow cytometry. J Neurosci Res 86:1944-1958.

Taoka Y, Okajima K, Uchiba M, Murakami K, Kushimoto S, Johno M, Naruo M, Okabe H, Takatsuki K (1997) Role of neutrophils in spinal cord injury in the rat. Neuroscience 79:1177-1182.

Tom VJ, Doller CM, Malouf AT, Silver J (2004) Astrocyte-associated fibronectin is critical for axonal regeneration in adult white matter. J Neurosci 24:9282-9290.

Vajkoczy P, Laschinger M, Engelhardt B (2001) Alpha4-integrin-VCAM-1 binding mediates $\mathrm{G}$ protein-independent capture of encephalitogenic $\mathrm{T}$ cell blasts to CNS white matter microvessels. J Clin Invest 108:557-565. 